Cahiers $d u$ MONDE RUSSE

\section{Cahiers du monde russe}

Russie - Empire russe - Union soviétique et États indépendants

46/1-2 | 2005

La Russie vers 1550

\title{
Le temporel de la chaire métropolitaine de Russia orientalis
}

(XIV ${ }^{\mathrm{e}}$ siècle -- premier quart du XVII ${ }^{\mathrm{e}}$ siècle)

\section{Élisabeth TEIRO}

\section{(2) OpenEdition}

\section{Journals}

Édition électronique

URL : https://journals.openedition.org/monderusse/8811

DOI : 10.4000/monderusse. 8811

ISSN : $1777-5388$

Éditeur

Éditions de l'EHESS

\section{Édition imprimée}

Date de publication : 1 janvier 2005

Pagination : 355-362

ISBN : 2-7132-2055-6

ISSN : $1252-6576$

Référence électronique

Élisabeth TEIRO, "Le temporel de la chaire métropolitaine de Russia orientalis », Cahiers du monde russe [En ligne], 46/1-2 | 2005, mis en ligne le 01 janvier 2007, consulté le 04 septembre 2022. URL : http://journals.openedition.org/monderusse/8811; DOI : https://doi.org/10.4000/monderusse.8811 


\section{CAIR N}

chercher : repérer : avancer

Cet article est disponible en ligne à l'adresse :

http://www.cairn.info/article.php?ID REVUE=CMR\&ID NUMPUBLIE=CMR 461\&ID ARTICLE=CMR 4610355

Le temporel de la chaire métropolitaine de Russia orientalis. (XIVe siècle -premier quart du xviie siècle)

par Élisabeth TEIRO

\section{| Editions de l'EHESS | Cahiers du monde russe}

\section{5/1-2 - Vol 46}

ISSN 1252-6576 | ISBN 2713220556 | pages 355 à 362

Pour citer cet article :

- TEIRO n, Le temporel de la chaire métropolitaine de Russia orientalis. (XIVe siècle -- premier quart du xviie siècle), Cahiers du monde russe 2005/1-2, Vol 46, p. 355-362.

Distribution électronique Cairn pour les Editions de l'EHESS.

(C) Editions de l'EHESS. Tous droits réservés pour tous pays.

La reproduction ou représentation de cet article, notamment par photocopie, n'est autorisée que dans les limites des conditions générales d'utilisation du site ou, le cas échéant, des conditions générales de la licence souscrite par votre établissement. Toute autre reproduction ou représentation, en tout ou partie, sous quelque forme et de quelque manière que ce soit, est interdite sauf accord préalable et écrit de l'éditeur, en dehors des cas prévus par la législation en vigueur en France. Il est précisé que son stockage dans une base de données est également interdit. 


\title{
LE TEMPOREL DE LA CHAIRE MÉTROPOLITAINE DE RUSSIA ORIENTALIS*
}

\author{
(XIV siècle - premier quart du XVII ${ }^{\mathrm{e}}$ siècle)
}

La notion de chaire métropolitaine a deux acceptions. Au sens large, elle désigne l'ensemble des diocèses placés sous l'autorité d'un métropolite ; au sens restreint, elle s'applique à l'éparchie particulière du métropolite qui, comme celles de ses évêques suffragants, possède une réalité territoriale, donc matérielle et temporelle.

Seuls les biens-fonds du diocèse des métropolites moscovites ${ }^{1}$ et certains aspects de leur gestion retiendront ici notre attention. Deux raisons ont motivé le choix de la tranche chronologique. Cette étude s'inscrit dans le cadre d'un travail plus large sur la chaire métropolitaine, de l'installation du métropolite Pierre (1308$\left.\dagger 1325 / 26^{2}\right)$ à Moscou (1325) à l'instauration du patriarcat (1589). Cette limite a été repoussée au début du XVII ${ }^{\mathrm{e}}$ siècle pour trois motifs. D'abord, les sources relatives à cette période sont très souvent éditées avec celles concernant les XIV $\mathrm{X}^{\mathrm{e}} \mathrm{XVI} \mathrm{I}^{\mathrm{e}}$ siècles. Ensuite, les sources de la fin du XVI et du début du XVII ${ }^{\mathrm{e}}$ siècle offrent des détails sur l'histoire des localités souvent absents des documents plus anciens. Enfin, elles enrichissent largement la liste des biens-fonds métropolitains; or il est très probable que l'écrasante majorité de ces terres était propriété de la chaire avant 1589.

\footnotetext{
* Par opposition à la Russia Occidentalis, la Russia orientalis désigne les territoires de la Russie qui, au cours de la période médiévale, ne furent jamais sous la domination de la grande-principauté de Lituanie. Sur cette expression, voir P. Gonneau, I. Sorlin, W. Vodoff, « Propositions pour une terminologie française du passé des slaves orientaux », Russia Mediævalis, IX (1), 1998, p. 5-12.

1. Je n'aborderai pas ici la question subtile du siège et de la résidence des métropolites de Russia orientalis à partir de Maxime (1287-†1305)

2. Sur le calcul de la date de la mort de Pierre, voir N. S. Borisov, Politika moskovskih knjazej : konec XIII-pervaja polovina XIV veka, M., 1999, p. 203-204.
} 
Le temporel métropolitain est un thème qui demeure peu exploré. M. I. Gorčakov eut certainement le tort de s'imposer des limites chronologiques trop larges (988$1738)^{3}$. La très sévère critique que fit $\mathrm{V}$. O. Ključevskij ${ }^{4}$ de son travail considérable n'encouragea peut-être pas d'éventuels disciples à l'exception majeure de S. B. Veselovskij ${ }^{5}$. Les publications actuelles de sources faciliteront les recherches de leurs successeurs.

Les sources spécifiques qui permettent d'étudier le temporel sont les testaments d'ecclésiastiques et de laïcs, les documents témoignant des transactions, les registres divers, à savoir les sources cadastrales (piscovye knigi), les livres de dons (vkladnye knigi), les pitanciers (kormovye knigi). Il faut aussi citer les textes polémiques sur les biens ecclésiastiques.

De nombreux problèmes méthodologiques apparaissent dès les premières recherches.

Il est quasiment impossible de connaître la composition et l'étendue du temporel métropolitain au début du XIV e siècle. Du fait de l'absence de sources, nous ne pouvons estimer ni le temporel de la chaire métropolitaine de sa création à l'installation de Maxime en Souzdalie (1299-1300), ni celui de l'éparchie de Vladimir qu'il accapara très certainement en s'asseyant sur le siège épiscopal de Siméon' ${ }^{6}$.

Établir l'inventaire des possessions métropolitaines constitue la deuxième difficulté avec laquelle il faut composer. Le temporel ecclésiastique se compose de biens immeubles et meubles et de revenus dérivant ou non de ceux-ci. S'il est ardu de dresser la liste des monastères et des terres, estimer le reste des possessions de la chaire métropolitaine est voué à l'échec. Pour cette raison, notre étude ne concerne que les terres, les monastères et la cathédrale de la Dormition à Moscou.

Définir les divisions administratives mentionnées dans les textes pose aussi problème. À travers l'espace géographique et le temps, d'un type de document à l'autre, un même terme désigne des réalités parfois radicalement différentes. Certaines sources témoignent du fait que les divisions administratives se superposaient ${ }^{7}$. Certains cantons urbains (stany) étaient constitués de deux zones non limitrophes ${ }^{8}$.

3. M. I. Gorčakov, O zemel’nyh vladenijah vserossijskih mitropolitov, patriarhov i sv. Sinoda : iz opytov issledovanija v istorii russkogo prava, $\mathrm{SPb} ., 1871$.

4. V. O. Ključevskij, Razbor sočinenija o. Gorčakova «O zemel’nyh vladenijah vserossijskih mitropolitov i patriarhov», M., 1873.

5. S. B. Veselovskij, Feodal'noe zemlevladenie Severo-Vostočnoj Rusi, M.-L., 1947.

6. Consacré en 1295 à la tête de la chaire de Vladimir, Siméon fut transféré à Rostov à l'arrivée de Maxime à Vladimir.

7. Dans deux actes du troisième tiers du XVI ${ }^{\mathrm{e}}$ siècle, le village Filippovskoe est localisé dans le district (uezd) de Perejaslavl', le canton urbain (stan) Borisoglebskij et le canton rural (volost') Marinina. De ces deux documents décrits par A. V. Antonov (RD, 2, № 193 (p. 105-106) et $\mathrm{N}^{\circ} 237$ (p. 111)), seul le premier a été édité par S. N. Kisterev ( $R D, 9, \mathrm{~N}^{\circ} 52$ (p. 148-152).

8. Voir la carte du district de Moscou établie par S. B. Veselovskij qui montre que les cantons urbains Ždanskij $\left(\mathrm{N}^{\circ} 17\right)$ et Ratuev $\left(\mathrm{N}^{\circ} 44\right)$ étaient disloqués, Istorija sel $i$ dereven' Podmoskov'ja, 4, M., 1993,p. 10-11. 
Localiser les biens-fonds est également difficile. Dans l'immense majorité des cas, cette tâche est tout à fait irréalisable du fait de l'absence complète d'indications dans les documents.

Si elles sont abondantes, les sources sont insuffisantes pour suivre le destin d'un bien. Même si nous faisions abstraction des difficultés de localisation, il serait impossible d'établir la carte des possessions métropolitaines pour une période donnée.

La chaire métropolitaine acquérait ses biens comme toutes les autres institutions ecclésiastiques.

Il faut d'abord citer l'accaparement du temporel d'éparchies (suffragantes), à commencer par celui du diocèse de Vladimir.

Du XIVe au début du XVII ${ }^{\text {e }}$ siècle, et en dépit des restrictions imposées par Ivan IV (1533-1547-†1584), la donation demeura une constante. Il n'en existait qu'un seul type : la franche aumône ${ }^{9}$ ( $v k l a d$ po duše) qui pouvait être assortie ou non de clauses multiples et contraignantes pour le bénéficiaire, destinées à limiter les abus. Ainsi que le souligne V. D. Nazarov, elle constituait une des sources les plus lucratives de l'accroissement du temporel ecclésiastique.

Les sources attestant la pratique de l'achat sont abondantes. Il n'était pas rare que le versement d'une somme en numéraire fût accompagné d'une soulte, à savoir du bétail sur pied ${ }^{10}$ ou des objets de valeur ${ }^{11}$. Si la chaire métropolitaine procédait à de nombreux achats, aucune vente de terre n'est attestée ${ }^{12}$.

L'échange était également une possibilité d'acquisition de biens-fonds. La chaire métropolitaine y recourait surtout avec des laïcs, beaucoup plus rarement avec d'autres institutions ecclésiastiques.

Enfin, la saisie d'un bien hypothéqué en garantie d'un prêt à crédit était une source d'accroissement du temporel métropolitain. Cette pratique prouve que l'Église se livrait à l'activité canoniquement proscrite de l'usure.

L'étude des sources montre que le noyau des possessions métropolitaines se trouvait centré sur le district de Moscou. Les types de localités acquises (villages, petits villages, hameaux) ne variaient pas probablement du fait que dans le district de Moscou (comme dans ceux de Dmitrov et Zvenigorod), la densité de population demeura aux $\mathrm{XV}^{\mathrm{e}-\mathrm{XVI}} \mathrm{I}^{\mathrm{e}}$ siècles plus forte que dans les autres régions. Les possessions dans les districts de Kostroma-Galič, Perejaslavl', Rostov et Vladimir étaient surtout représentées par des hameaux. A l'ouest et au nord-ouest, la chaire métro-

9. «Franche (ou pure) aumône : don à un établissement religieux en échange de prières et de messes en faveur du donateur », in F.-O. Touati, éd., Vocabulaire historique du Moyen âge, P., 1995, p. 90

10. Le 4 avril 1518, le dvoreckij Fedor F. Surmin achète à Afanasij et Aleksej M. Čertovy le petit village Ratislavle 120 roubles et une vache, $A F Z H, 1, \mathrm{~N}^{\circ} 74$.

11. Le 9 avril 1526, le $d v o r e c k i j$ Rusin F. Fomin verse à Jurij B. Perepečin 500 roubles et une pelisse de martre pour les localités qu'il lui achète dans le district de Suzdal', $A F Z H, 1, \mathrm{~N}^{\circ} 161$.

12. Nous ne pensons pas que la question du choix des documents à conserver entre ici en ligne de compte. 
politaine acquit le village Vvedenskoe-Dymcova et ses 31 hameaux (1568-1569)13. Probablement au XVI ${ }^{\mathrm{e}}$ siècle, elle devint propriétaire de localités dans les districts de Kašin ${ }^{14}, \mathrm{Klin}^{15}$, Starica ${ }^{16}$ et Zubcov ${ }^{17}$, c'est-à-dire sur le territoire de l'ancienne principauté de Tver'. Il faut noter que tel ne fut pas le cas dans les limites de la république de Novgorod, à l'exception des possessions de Bežeckij Verh. Mais cette région était passée sous domination moscovite dès le règne de Vasilij Irr (13901425). De même, nous ne trouvons pas trace de biens-fonds métropolitains dans les principautés de Jaroslavl' et de Rjazan'. Les raisons de cet état de fait demeurent pour nous inconnues. Les possessions de la chaire se situaient dans les mêmes districts que les monastères métropolitains ${ }^{18}$. Même s'ils ne géraient pas nécessairement les biens-fonds dans leur district, ils servaient peut-être de relais pour les administrateurs en titre. Or, il n'y avait pas de monastères métropolitains à l'ouest, au nord-ouest de Moscou ni dans les principautés de Jaroslavl' et Rjazan'. Si cette hypothèse était fondée, elle pourrait expliquer pourquoi, à en croire les sources, la chaire métropolitaine ne chercha pas à accroître son temporel dans les zones où elle n'avait pas de fondation. Mais un fait resterait inexpliqué : pourquoi ne reçut-elle pas, à Jaroslavl' et à Rjazan', de donations en franche aumône comme celles qui lui permirent de constituer son temporel dans l'ancienne principauté de Tver' ?

Une des méthodes d'exploitation par la chaire métropolitaine de ses biens-fonds était leur octroi en bénéfice (pomest'e).

Le système métropolitain des bénéfices était similaire au système grand-princier, à cette exception majeure que la métropole ne rétribuait pas un service militaire ${ }^{19}$. Autant que les sources permettent d'en juger, la chaire métropolitaine rémunérait ainsi les membres de sa cour (bojare et bojare cadets), ses autres

\section{3. $A R G, 1, \mathrm{~N}^{\circ} 3$.}

14. Les sources concernant le district de Kašin témoignent des années 1589-1590 - 15951596, AFZH, 3/2, $\mathrm{N}^{\circ} 23$ et 79.

15. À l'exception de la donation de deux villages (Kozinskoe et Sesi-Petrovskoe) faite par la princesse Elena Ol'gerdovna et qui date des années 1430, les acquisitions (toutes en franche aumône) de la chaire métropolitaine dans le district de Klin remontent aux années 1571-1572 - 1578-1579, DDG, $\mathrm{N}^{\circ} 28 ; R D, 4, \mathrm{~N}^{\circ} 33$ (p. 102-104) et $\mathrm{N}^{\circ} 35$ (p. 106).

16. Deux donations en franche aumône au monastère du Miracle sont attestées entre 1571 et $1573-1574, R D, 2, N^{\circ} 270$ et 271 (p. 115).

17. Les sources témoignent d'acquisitions régulières, particulièrement par donation, entre $1521-1523$ et $1578-1579, D D G, \mathrm{~N}^{\circ} 99 ; R D, 2, \mathrm{~N}^{\circ} 283$ (p. 116).

18. Il faut mentionner ici l'exception du monastère Saint-Nicolas Venev de Tula, fondé avant 1407. Je n'ai trouvé aucune source concernant le temporel de cette fondation recensée par M. I. Gorčakov comme métropolitaine.

19. Les membres de la cour métropolitaine étaient potentiellement soumis à l'obligation de service militaire dans les armées du grand-prince (en vertu de l'accord passé entre Vasilij Irr et Cyprien, $A S E I, 3, \mathrm{~N}^{\circ} 6$ ). S. B. Veselovskij a montré qu'en 1535 un régiment entier de membres de la cour métropolitaine fut décimé par les Lituaniens lors d'une bataille près de Starodub, S. B. Veselovskij, Issledovanija po istorii klassa služilyh zemlevladel'cev, M., 1969, p. 249 ; id., Feodal'noe..., op. cit., p. 428. Cette réalité est encore attestée à l'époque d'Andrej Kurbskij $\left(P L D R, 5\right.$, p. 364) et au début du XVII ${ }^{\mathrm{e}}$ siècle $\left(A F Z H, 1, \mathrm{~N}^{\circ} 192\right)$. 
fonctionnaires (comme les secrétaires), plus rarement ses monastères ${ }^{20}$ et des paysans ${ }^{21}$.

Les bénéficiaires de la chaire métropolitaine étaient parfois confrontés aux mêmes difficultés que ceux des grands-princes (et tsars) : ils devaient eux-mêmes chercher les terres auxquelles leur dotation leur donnait droit ${ }^{22}$. Pour comprendre cette situation, deux explications peuvent être avancées : soit c'est l'efficacité de la lourde administration métropolitaine qui est en cause ${ }^{23}$, soit la chaire métropolitaine menait une politique délibérée de non-attribution des terres à ses ayants droit, d'autant plus qu'elle devait rémunérer sur ses biens-fonds propres des serviteurs des tsars ${ }^{24}$. La réalité tenait certainement des deux. En effet, s'il est difficile d'estimer la population des bénéficiaires ainsi que la superficie totale des possessions métropolitaines, force est de constater que les surfaces accordées laissent entrevoir que le patrimoine de la chaire métropolitaine était suffisamment vaste pour rémunérer tous ses fonctionnaires. D'autant plus que les surfaces octroyées n'étaient pas uniquement des terres arables ou labourées. De nombreux exemples montrent que la chaire attendait qu'une terre se libère pour y installer un nouveau bénéficiaire alors qu'elle aurait pu lui octroyer la même surface ailleurs ${ }^{25}$.

Il semble que les serviteurs métropolitains, bénéficiaires en puissance, doivent être considérés comme des agents potentiels et nécessaires de l'exploitation du temporel métropolitain. Tous les bénéficiaires devaient s'engager à ne pas aliéner les terres qui leur étaient accordées souvent à perpétuité. Certains étaient obligés par contrat d'exploiter le domaine alloué.

Des documents de la fin du XVIe siècle et du début du suivant montrent clairement que l'octroi d'un bénéfice métropolitain était conditionné non seulement par le service de la chaire métropolitaine, mais aussi par celui du tsar. Dans ces sources, les formules adoptées ne font pas référence au service militaire mais au « service(s) du tsar $»^{26}$. Les pratiques attestées par ces documents doivent donc être considérées

20. Voir par exemple l'octroi du lac Kodlivoe à Pacôme, higoumène du monastère Saint-Côme (11 mars 6985/1477) puis à Matthieu, celui du monastère Saints-Constantin-et-Hélène (7 février 7019/1511), AFZH, 1, N²38, p. 241.

21. Le 18 mars 1527, Daniel accorde en bénéfice au paysan Dementij N. Novikov plusieurs localités dans le district de Vologda, $A F Z H, 1, \mathrm{~N}^{\circ} 314$.

22. Voir par exemple, $A F Z H, 3 / 2, \mathrm{~N}^{\circ} 6,10$ et 35 .

23. La comparaison des sources montre clairement que, dans de nombreux cas, l'administration métropolitaine n'utilisait pas les documents cadastraux les plus récents. Par exemple, un cadastre du canton de Bykov (district de Moscou) avait été réalisé par Vasilij Tihonov en 15881589. Or, le bénéfice concédé en 7104/1596 à Boris N. Turgenev l'est sur la base du cadastre établi par Grigorij Boltin et Evrej Semjagin en 1466-1467, AFZH, 3/2, No 58, p. 91.

24. Voir infra.

25. Voir par exemple $A F Z H, 3 / 2, \mathrm{~N}^{\circ} 100$

26. “А Констентину с того поместья государева и великого князя Бориса Федоровича всеа Руси служба служити”, $A F Z H, 3 / 2, \mathrm{~N}^{\circ} 192$. Avant 1614-1615, Tret jak Sobolev affirme “А служу, государь [Иона митрополит Крутицкий — E.T. ] с того поместейца я всякие царьские службы [...]”, ibid., $\mathrm{N}^{\circ}$ 263. Si la formule au singulier peut laisser planer un doute, tel n'est pas le cas du pluriel, d'autant plus que l'adjectif всякие insiste sur la diversité des services rendus. 
comme une forme d'accaparement du temporel métropolitain par le pouvoir séculier. Il s'agissait d'une méthode certainement plus rentable que d'essayer de limiter les acquisitions et les privilèges ecclésiastiques.

Le temporel métropolitain était considérable. Aussi pouvons-nous nous étonner que seuls 33 documents témoignent de différends relatifs aux biens-fonds. La question des principes d'archivage des actes réapparaît ici. En effet, ces 33 sources ne rapportent que des décisions favorables à la chaire métropolitaine. Nous n'avons, par exemple, pas trouvé dans les documents métropolitains le jugement de Vasilij I ${ }^{\text {er }}$ en faveur de la Trinité-Saint-Serge dans le conflit qui la mit aux prises avec un pêcheur métropolitain ${ }^{27}$.

Les différends qui opposaient les fondations et les serviteurs de tous rangs de la chaire métropolitaine à des monastères métropolitains et non métropolitains, aux princes et grands-princes et leurs serviteurs, à des paysans taillables étaient surtout territoriaux. Les nombreux documents cadastraux l'attestent tout autant que les minutes des procès.

Dans la mesure où il n'y a pas de différence entre les règlements des conflits impliquant la chaire métropolitaine ou d'autres institutions ecclésiastiques, nous mettrons l'accent sur quatre points.

Premièrement, quelles qu'aient été les parties prenantes d'un différend, il était jugé par une autorité laïque. Cela constitue une preuve supplémentaire du statut ambigu des terres en Russie.

Deuxièmement, les litiges entre deux fondations métropolitaines ${ }^{28}$ soulignent l'autonomie économique de ces sous-ensembles au sein de l'éparchie métropolitaine. Les enjeux, qui nous paraissent négligeables, étaient loin de l'être pour les moines qui n'hésitaient pas à régler leurs conflits devant une justice laïque. Si les conséquences des jugements étaient sans doute neutres pour la chaire métropolitaine qui imposait ses fondations, elles prenaient une autre dimension à l'échelle de ces monastères. La perte ou le gain d'une zone exploitable étaient synonymes de diminution ou d'augmentation de revenus et, éventuellement, du volume de la main-d'œuvre, dont la valeur intrinsèque était plus importante que celle de la terre.

Troisièmement, la chaire métropolitaine ne faisait pas exception : comme de nombreux monastères ${ }^{29}$, notamment, elle cherchait à étendre son temporel dans des zones non taillables.

Quatrièmement, dans la mesure où nous ne pouvons suivre le destin des biensfonds métropolitains, il est impossible de dire si les décisions rendues étaient toutes justes. Si tel n'était pas le cas, il faudrait considérer ces jugements quasiment comme des donations à la chaire métropolitaine.

27. Voir P. Gonneau, La Maison de la Sainte-Trinité : un grand monastère du Moyen Âge tardif, 1345-1533, P., 1993, p. 313.

28. RD, 2, $\mathrm{N}^{\circ} 120$ (p. 196) et $A F Z H, 1, \mathrm{~N}^{\circ} 208$.

29. Voir, par exemple, I. U. Budovnic, Monastyri na Rusi i bor'ba s nimi krest'jan V XIV-XVI vV., M., 1966 ; P. Gonneau, La Maison..., op. cit., p. 322-338. 
Le volume et l'exploitation du temporel métropolitain révèlent les ambiguïtés de la gestion des terres. D'une part, la superficie des terres crût en permanence, provoquant l'augmentation des effectifs de la cour métropolitaine nécessaires pour les administrer. La chaire métropolitaine semblait pourtant hésitante à les leur distribuer. De manière générale, l'organe central de gestion faisait preuve de réticence à l'idée de déléguer la gestion de son temporel. D'après S. B. Veselovskij, les monastères métropolitains administraient seulement un tiers du volume total des biens-fonds. Si des monastères métropolitains furent fondés durant les $\mathrm{XV}^{\mathrm{e}}$ $\mathrm{XVI}^{\mathrm{e}}$ siècles, ils demeurèrent des structures microscopiques. Aussi pouvons-nous nous demander pourquoi la chaire métropolitaine ne fonda pas dans les zones de grande concentration de ses biens-fonds des monastères suffisamment puissants pour l'assister dans la gestion de ses terres. D'autre part, la chaire métropolitaine n'échappa pas au problème endémique de l'agriculture en Russia : le manque de bras. En dépit des avantages que présentait, pour un paysan qui en avait le droit, la possibilité de s'installer sur les terres de l'Église, il est évident que les terres métropolitaines étaient largement sous-exploitées. Aussi est-il curieux de constater qu'à l'instar de toutes les autres institutions ecclésiastiques, la chaire tendit en permanence à accroître ses possessions, non seulement en acquérant de vastes domaines, mais aussi en rongeant les zones taillables comme si elles représentaient pour elle un enjeu vital.

Si les méthodes de gestion du temporel métropolitain peuvent paraitre inadaptées, il faut reconnaître qu'elles firent leurs preuves et furent suffisamment efficaces pour conserver à la métropole une puissance économique considérable que le pouvoir séculier sapera beaucoup plus lentement que sa puissance politique.

École pratique des hautes études

IVe section : Sciences historiques et philologiques

elisabeth.teiro@club-internet.fr 